\title{
Using Explicit Control Processes in Distributed Workflows to Gather Provenance
}

\author{
Sérgio Manuel Serra da Cruz ${ }^{1}$, Fernando Seabra Chirigati ${ }^{1}$, Rafael Dahis ${ }^{1}$, \\ Maria Luiza M. Campos², and Marta Mattoso ${ }^{1}$ \\ ${ }^{1}$ PESC - COPPE \\ ${ }^{2} \mathrm{PPGI}-\mathrm{IM} / \mathrm{NCE}$ \\ Federal University of Rio de Janeiro (UFRJ) \\ P.O. Box: 68511, Rio de Janeiro, RJ, 21941-972, Brazil \\ \{serra, fernando_seabra, rafaeldahis, marta\}@cos.ufrj.br, \\ mluiza@ufrj.br
}

\begin{abstract}
Distributing workflow tasks among high performance environments involves local processing and remote execution on clusters and grids. This distribution often needs interoperation between heterogeneous workflow definition languages and their corresponding execution machines. A centralized Workflow Management System (WfMS) can be locally controlling the execution of a workflow that needs a grid WfMS to execute a sub-workflow that requires high performance. Workflow specification languages often provide different controlflow execution structures. Moving from one environment to another requires mappings between these languages. Due to heterogeneity, control-flow structures, available in one system, may not be supported in another. In these heterogeneous distributed environments, provenance gathering becomes also heterogeneous. This work presents control-flow modules that aim to be independent from WfMS. By inserting these control-flow modules on the workflow specification, the workflow execution control becomes less dependent of heterogeneous workflow execution engines. In addition, they can be used to gather provenance data both from local and remote execution, thus allowing the same provenance registration on both environments independent of the heterogeneous WfMS. The proposed modules extend the ordinary workflow tasks by providing dynamic behavioral execution control. They were implemented in the VisTrails graphical workflow enactment engine, which offers a flexible infrastructure for provenance gathering.
\end{abstract}

Keywords: workflow, provenance, scientific workflow, distributed computing.

\section{Introduction}

Scientific experiments are increasingly being conducted using workflow specifications controlled by workflow management systems (WfMS). Obtaining provenance data for scientific experiments with the help of these systems is becoming very attractive. However, provenance capture mechanisms are still an open issue [1]. Innumerous WfMS are available to support scientific workflow execution [1] [2] [3]. Each system has specific features to address different workflow requirements. It is very 
likely that workflows belonging to the same experiment use different WfMS. Each WfMS has its own provenance model and capture mechanism. Such heterogeneity imposes many challenges for provenance data representation and integration. Several efforts are underway to minimize this heterogeneity, such as the Open Provenance Model (OPM) [4] and the series of Provenance Challenges.

Scientific workflows are characterized by data intensive analyses. These data driven workflows also need control structures to specify how the data flow should be directed [5] [6] [7]. Often, these data analyses are time consuming and need to execute on remote parallel processing environments, such as cluster machines and grids. Several grid WfMS [3], such as Pegasus [8], P-GRADE [9] and Triana [10], have been proposed to offer high performance execution. However, not all tasks of a scientific workflow need to be executed on a high performance environment. Local WfMS have been designed with rich graphical interfaces to be interactively used. For example, they provide visual tools to follow the workflow execution steps. Local and centralized control based WfMS (e.g. Taverna, [11], VisTrails [12] [13], and Kepler [14]) are characterized by a single workflow execution engine, enacting the whole execution of a given scientific workflow.

A distributed workflow is characterized by a distributed execution where some of the workflow tasks are best executed locally, while others remotely. This is a typical scenario for many applications, such as bioinformatics. Local task execution is well supported by current centralized WfMS. However, they present limited capabilities to allow for remote executions. Usually, the WfMS presents some modules that help the invocation of a remote execution environment for executing one single task. When it is necessary to run a sub-workflow remotely, WfMS language mappings are required. Using one single workflow language/engine to run tasks of the same workflow along these local and distributed environments is currently an open issue.

In summary, a distributed workflow execution involves a lot of heterogeneity, i.e., execution under different workflow engines and heterogeneous provenance capture mechanisms. Provenance gathering based on central control can lose track of these remote tasks. Provenance gathering is usually tightly coupled to the workflow engine in charge of execution monitoring. Moving from one workflow system to another imposes at least two challenges. The first is related to the workflow design - in general, scientists are focused on composing and constructing a particular workflow within one single WfMS; designing parallel executions of processes may involve heterogeneous workflow models. The second relates to provenance gathering - currently, most WfMS register provenance in their own schema, often encompassing specific grid features or application domain attributes. Even if all systems adopt the same provenance schema, e.g. OPM [4], integrating its instances is not trivial.

One possible solution is to diminish the dependence of the workflow definition on the WfMS. This could be achieved by uncoupling the provenance gathering system from the WfMS, and also by having some control-flow of execution independent of the WfMS workflow specification language. By plugging control-flow and provenance gathering modules along the workflow original tasks, the workflow specification can be executed almost independently of the current WfMS and provenance can be gathered uniformly.

In this paper, it is proposed a small set of generic workflow-level control modules that can be used to design a scientific workflow to be executed by heterogeneous 
WfMS. These control modules are close to basic workflows patterns proposed in [15]. They aims to improve remote workflow execution by allowing WfMS to face a dynamic execution behavior without losing track of the provenance gathering process in distributed environments. Such approach is a step towards the above-mentioned challenges on independent distributed workflow execution and provenance gathering.

This paper is organized as follows. Section 2 discusses scientific workflow controls and distributed provenance gathering. Section 3 presents the execution control modules. Section 4 shows an implementation of these modules on VisTrails WfMS. Section 5 concludes the paper.

\section{Provenance Gathering in Distributed Scientific Workflows}

In this section, we discuss the problems of having to move from one WfMS to another. Initially, we stress that control flow does matter in scientific workflows. Then, we discuss the problems of having different control structures in WfMS specification languages and address its impact on provenance gathering.

\subsection{Control Flow in Scientific Workflows}

Even though control is not the main characteristic of a scientific workflow, they do matter and are essential in many experiments [5] [3] [6] [7]. Workflow control patterns have been proposed by Aalst et al. [16] as a common framework to help on the heterogeneities of the several workflow specification languages. We used a subset of these control-flow patterns to design our proposed control modules. Our goal is to have a meta-workflow specification language that eases migration from one WfMS to another.

According to the taxonomy of Yu and Buyya [3] for Scientific Workflow Systems for Grid Computing, a DAG-based workflow structure can be categorized as sequence, parallelism, and choice, whereas a scientific non-DAG workflow also includes iteration structure. Control structures play an important part on this taxonomy that represents generically scientific workflows

Goderis, Brooks, Altintas, Lee, and Goble [6] stress the importance of combining different models of computation in one scientific workflow. They show examples of combining sequential pipelines with iteration and choice control flow process similar to a finite state machine that evaluates guards on all outgoing transitions. Similarly, Bowers, Ludaecher, Ngu and Critchlow [5] say that control-flow modeling, such as "ifthen-else and switch-case statements, and iteration with multiple entry and exit points", are often necessary for engineering fault-tolerant, robust, and adaptive workflows. They also say "that modeling control-flow using only dataflow constructs can quickly lead to overly complex workflows that are hard to understand, reuse, reconfigure, maintain, and schedule." They present a similar approach to our proposal. They have designed a set of templates to represent control structures as actors in the Kepler WfMS [14]. Our approach differs from [5] and [6] in the sense that they present control structures for one specific WfMS, while we are focusing on general control-flow structures to simplify sub-workflow remote execution. In addition to reuse, we aim at gathering remote process provenance with the help of these control structures. 
Tudruj, Kopanski and Borkowski [7] also state the importance of general dynamic control flow, but focus on synchronization of parallel execution. Similarly to our proposal they have presented a set of generic control structures and proposed the use of a monitoring middleware. However, we do not want to interfere on the execution of tasks on a remote distributed environment. So, our focus is more on remotedistributed provenance gathering rather than synchronization control.

\subsection{Provenance Gathering in Heterogeneous WfMS}

WfMS have been designed as distributed or centralized execution control. While distributed WfMS, like Pegasus [8], P-GRADE [9] and Triana [10], focus on high performance and resource scheduling, rather than provenance gathering, centralized control based WfMS (e.g. Taverna, [11], VisTrails [12] [13], Kepler [14]) are focused on semantic issues in workflow design and provenance gathering. However, centralized WfMS are characterized by having a single workflow execution engine, enacting the whole execution of a given scientific workflow. For instance, VisTrails provides visualization facilities and provenance of the whole exploration process, capturing the evolution of a workflow. Despite these facilities, VisTrails, in its current public version, lacks support in connecting to distributed environments and does not allow inner activities loops. Kepler has some predefined control-flow modules that can be plugged in the workflow specification. However, if a sub-workflow needs to be executed under a different WfMS, these Kepler's actors will no longer apply.

If you need to change from one environment to another, from local execution to a grid, you may send tasks of a sub-workflow to be remotely executed one by one. In this way, you can keep the local WfMS in charge of the execution control, but this can deteriorate performance severely. Another option is to recode the sub-workflow with the grid WfMS language, then this sub-workflow will execute under the grid WfMS and take advantage of the remote grid resources without coming back and forth to the local WfMS. Alternatively, if you send general control modules along the sub-workflow, you may send this augmented sub-workflow to run remotely and still be able to register provenance remotely and bring it back locally. Otherwise, if you rely on the provenance system of the remote WFMS, you will need to do conversions from one provenance model to another, and you may miss some provenance gathering not supported by the remote system and so on.

In this scenario, a typical scientific workflow may want to have its provenance recorded by a centralized WfMS, as well as to take advantage of high performance environments, such as the ones provided by grid WfMS. We aim at showing that having control independent from the WfMS gives flexibility to help in moving from one WfMS to another in a distributed environment, e.g. local and grid. These additional modules can further be used to record provenance remotely using the same representation model as the local WfMS, or OPM for example.

Having control modules explicitly defined can help on other workflow semantic issues. When control-flow specifications are based on patterns (standards), reusing parts of a workflow becomes easier. If control modules have a formal basis, several verifications can be done. For example, does the workflow terminate? Does it conform to some correction rules? This can lead to a meta-workflow definition. It can be 
seen as a step towards a canonical model or a layer to where all workflow languages and provenance models can be mapped to and from.

\section{Scientific Workflow Control Flows}

In this section, we present our approach to provide scientific workflows with generic control-flow modules for the coordination of the traditional data flow operations. These structures enable the execution of distributed applications with scheduling dependencies more advanced than the simple data availability criteria, and where the data passing between activities need to be restricted due to processing errors, or data volume, or costs associated with transfers. Aalst et al. [15] [16] have identified many patterns that provide a systematic examination of the various perspectives that need to be supported by workflow languages. In short, the control-flow perspective captures aspects related to control-flow dependencies between various activities of a workflow. The data perspective deals with the information flow and variables scope, among others. The resource perspective deals with resource to activity allocation and delegation. Finally, the patterns for the exception handling perspective deal with many causes of exceptions and the consequent actions needed to overcome them.

This work is centered on the control-flow perspective, because control patterns define which activities of a given workflow should become enabled after the completion of other activities and in what order they will be executed by the scientific WfMS. It is also important to highlight that the control patterns are not concerned with how an enabled activity will be executed and whether it works as it is supposed to do. The control flow pattern considers each activity as a black box and the only observable behavior which the pattern is concerned about is when the activity becomes enabled and when it finishes executing.

WfMS like Taverna [17], Kepler [14] and VisTrails [12] [13] present a heterogeneous and limited set of control-flow elements, making it difficult to design a scientific workflow which often requires iterations, decision, conditions and registering of processes' data. One of the possible ways to bypass those shortcomings requires reprogramming the WfMS. However, modifying existing WfMS may be time consuming and error prone. Another way is to reuse generic control-flow modules, such as the ones we propose in this work. Table 1 shows these modules, the number of input/output ports and the corresponding workflow pattern as defined by Aalst et al. [16].

Table 1. Control flow modules x workflow patterns

\begin{tabular}{lcl}
\hline Module & Number of input/output ports & Workflow pattern \\
\hline Mux & 1 (selector) + user defined / 1 & Structured Discriminator \\
Demux & $2 /$ user defined & Exclusive Choice \\
String Control & $2 / 2$ & Deferred Choice \\
NumberControl & $1 / 5$ & $\begin{array}{l}\text { Multiple Instances Without syn- } \\
\text { chronization }\end{array}$ \\
& $2 / 1$ & Synchronization \\
NumberCompare & $4 / 1$ & Exclusive Choice \\
If & &
\end{tabular}


The proposed workflow controls are extensible components that allow the workflow to be redefined at run-time, offering it the ability to adapt automatically to changes without compromising the logic of the scientific experiment and its safety. These control modules can be embedded in a graphical workflow design interface, enabling workflow programming with run-time modifiable functionality and dynamic interactions between activities. Thus, the workflow tasks can move from one workflow engine to another without having to recode the workflow specification to match heterogeneous workflow specification languages. In addition, when control moves, provenance gathering can move along and be aware of the remote execution flow. This flexibility comes with some limitations, for example, the workflow execution scheduler is not aware of control flow and may provide poor optimization. However, when specific workflow coding prevents provenance gathering, trading performance might be an option. In the next section, we present the control flow modules and compare them with workflow patterns.

\subsection{Control Flow Modules in VisTrails}

In this work, we used the VisTrails WfMS [12] [13] to implement the proposed control-flow modules by adding dynamic behavior without harnessing the provenance gathering process of the WfMS. In VisTrails, the workflow tasks are known as modules and are coded in Python. VisTrails presents some implementation requisites, such as: (i) each module is defined as a class, which describes a given structure; (ii) modules are connected through input/output ports; (iii) input values come through input ports, and results are given in output ports; and (iv) all the connection and relationship between the modules must be defined and formalized inside the class. Modules in VisTrails, as in other WfMS, like Kepler [14], require a data type. Developers need to know the type of the data that are passing through them, despite the programming language used in their code. Thus, each port must have a defined type (e.g. string, float, integer, file or boolean). Modules with similar goals can be grouped as packages in order to simplify deploy and development, making their organization clearer.

\subsubsection{Multiplexer and Demultiplexer}

The Multiplexer module, or simply Mux, is important for decision making activities. Its functionality lies on the choice of a data piece, between a series, to continue in the execution flow. Mux module offers the possibility of choosing an input port; such feature is not directly available in VisTrails or in other WfMS. The pattern that best represents the Mux module is the "Structured Discriminator" [15]. This pattern describes a convergence between two or more input ports, resulting in just one branch. The activation of the output port depends on an input port, what happens in the Mux module. However, in the module, there is a condition (the selector) to choose the input port; while in the pattern, the first incoming branch to be enabled is the one to activate the outgoing branch; it does not depend on a condition. Besides, in the pattern, there must be a "Parallel Split" pattern before, which not necessarily happens with the module. In VisTrails, the Mux module implementation has two user-defined characteristics: the number of input ports and the data type required. Both of them increase the flexibility of the activity. 
The Demultiplexer module, or Demux, does the opposite of Mux. It selects the path that a piece of data will take. In other words, it makes the decision of what subsequent part of the workflow will get a piece of data and process it. It is important to notice that the same feature presented by the Mux module (the selection of desired ports) is also created by the Demux module. This module would be an example of the "Exclusive Choice" pattern [15]. This pattern represents an incoming branch that diverges into two or more parts. Just one of the outgoing branches is enabled depending on a condition associated. This condition, in the Demux module, would be the selector, which chooses the output port.

The Demux module, in VisTrails, has only one input port for the incoming data, and another for the selector. Its implementation was done in the same way as for the Mux module; the difference between them is that, instead of choosing the number of input ports, in Demux the user chooses the quantity of output ports.

There is one problematic situation associated to this pattern which is related to the structure response when the condition does not match any of the output ports. In VisTrails, the Demux module displays a warning message when a condition is reached, and all the execution stops. Besides, there is no way more than one outgoing branch to be enabled, once the selector is an integer, and each integer corresponds to only one branch.

\subsubsection{String and Number Controls}

In some cases, the workflow needs to generate control signals produced through comparison of strings or numbers. A control signal behaves as a flag for the execution of other activities. For that reason, such controls are particularly important and may be considered as control structures. The flags are usually used for decision making; so, they can be used in scientific workflows for the same purpose. The StringControl module represents the "Deferred Choice" pattern [15]. In this pattern, an activity in the workflow is divided in two or more branches, and just one of them can be enabled; the other outgoing branches are withdrawn. StringControl module acts like such pattern, only one of the output ports can be enabled. However, there is just one difference between the StringControl module and the pattern, in the latter, the faster outgoing branch to make an activity is the one which will be enabled; in the module, it depends on the number of input ports connected.

In VisTrails, StringControl has two input ports to receive strings. The StringControl execution depends on the number of strings connected. If there is just one, the module will return the length of it by an output port (an integer value). However, if two strings are connected, the module will do a comparison between them returning " 1 " for greater, " 2 " for smaller and " 0 " for equal strings.

For number signals, two new modules were created. The first one, NumberControl, generates flags that indicate whether the number is zero, negative, positive, odd or even. These signals can be essential if a given workflow activity depends on the number type. The second one, NumberCompare, on the other hand, compares two numbers, signaling which one is greater or smaller, or if both are equal. These signals are similar to the ones generated by StringControl module.

The NumberControl module is correlated with a variant of the "Multiple Instances without Synchronization" pattern [15], where all output data are originated simultaneously. The NumberCompare module, in the other hand, represents the "Synchronization" 
pattern [15]. In this pattern, two or more incoming branches become just one outgoing branch, which will be only enabled after the complete activation of all the input data. In other words, there must be a synchronization between the incoming branches. This is what happens with the "NumberCompare" module, once the comparison between the numbers can only be done with both of them enabled in the module.

\subsubsection{If}

The use of conditionals are particularly important when scientists need to orchestrate data flows, specifying which will be the next data flow to run based on the control decision, such as: knowing if an expression is true, choosing data between two input ports, or for stopping a whole workflow execution. The pattern that represents the If module is the "Exclusive Choice" pattern [15], the same pattern of the Demux module. The two essential differences between the Demux module and the If module are: in the former, the scientist can connect as many input ports as they need, different from the latter, which has just two input ports; in the Demux, there is an integer selector to choose between many input ports, and in the If module the selector is a logical expression, where the scientists can create any condition they need.

Its implementation in VisTrails has four input ports: two of them are the data that will be chosen, which can be of any type supported by VisTrails. Another port is used to write the condition associated to the module: if the condition is true, the first data is passed through the output port; if false, the second one is chosen. The last input port, which is optional, is an extra code that the users can write before the condition; maybe, for the desired condition, they need to import some libraries, make some logical and arithmetic expressions, read from a file, and so on; for this kind of programming, they can use this input port. The only output port is the chosen data.

There is another use for the If module. The second input port is optional; if the user chose to put that port, the functionality of the module will be the one stated before. However, if there is no second input port, the module will put the first input port in the output port if the condition is true; if false, the module will raise an error, stopping the workflow execution.

\subsubsection{Inner Loops}

It is important for a given workflow to have a sequence of commands to be executed repeatedly. It means that the workflow will execute an activity several times in a cyclic execution. This type of sequence is called inner loop, and it is useful for some loop cases. The loops inside a module could be done using a programming language. However, the loop encompassing several modules cannot be done so easily for some WfMS, particularly DAG-based. For instance, VisTrails does not allow recursion in the workflow. But it offers a mechanism named parameter exploration, which allows a scientist to make repetitive executions of a given scientific workflow, using different parameters for the input ports. The loop originated through the parameter exploration represents the "Multiple Instances with a priori Run-Time Knowledge" pattern [15]. In this pattern, series of multiple instances of an activity are created, and the number of instances is known before the process starts. This is precisely what happens with the loop in VisTrails. The instances run concurrently and they are created sequentially. 
In this way, a kind of loop is created, since all activities of the workflow are reinitialized several times. However, there are two crucial differences between this kind of loop and the real one. The first one is that the workflow, in the parameter exploration, is finalized before it returns to the beginning, what does not occur in the loop itself. The second one is related to the call of the loop; in some cases, the loop must be called inside the workflow, and not outside of it, as it is with the VisTrails feature.

In order to offer simple but effective inner loop specification, it is possible to use a composition of control flow modules to execute an inner loop in VisTrails. For instance, a scientific workflow may need to process five different files, each one in a separated execution. One feasible way to make an inner loop with control modules rely on the use of the Mux module and the Register, a module that writes the data in a pre-defined file. At first, the scientist has to define the number of input ports of the Mux and its data types. After that, the files can be easily connected to the Mux ports, and its output port can be connected to the Register module. The parameter exploration is used, in this case, to generate a series of workflow executions by changing the Mux selector and for each execution, the file processed in the subsequent part of the workflow is replaced by other file connected to the Mux through a user-defined linear interpolation of the selector, for example (Figure 1). So, the workflow will be executed for each value inside the interpolation.

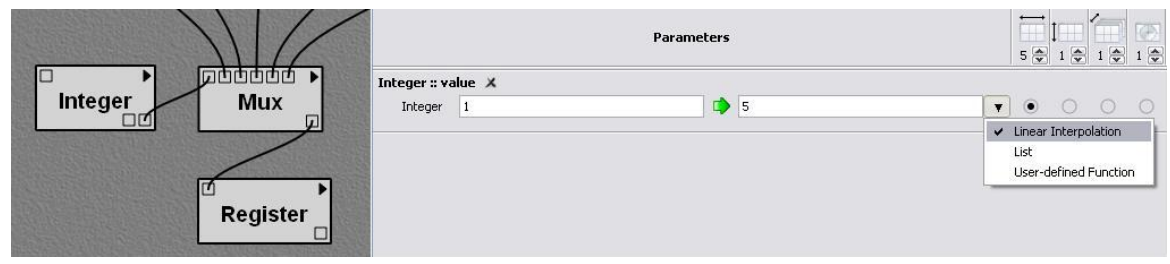

Fig. 1. VisTrails screenshot with control flow modules. On the left side, a part of the workflow is presented (the Integer module represents the selector); On the right side, using VisTrails' parameter exploration, a linear interpolation with the selector values is shown, and it generates an inner loop.

\section{Execution Control on VisTrails}

Due to modularity and flexibility of VisTrails open source WfMS, our execution control modules were easily added to VisTrails. In Figure 2 we highlight the execution control modules incorporated in VisTrails through its interface. Preliminary tests showed the design power of including execution control previously not available in VisTrails. A bioinformatics workflow that requires execution control modules can now take advantage of VisTrails process provenance and its visual resources. In order to combine the control-flow modules, presented in Section 3, with remote parallel execution control, a parallel bioinformatics workflow was evaluated (Figure 2).

In a previous work [18], we designed a similar, but simpler, bioinformatics workflow using Kepler WfMS. This bioinformatics workflow needs execution control such as If and NumberControl as well as remote parallel execution of Blast. The lack of remote parallel execution control in most WfMS motivated the development of our monitoring middleware named MidMon [18]. 


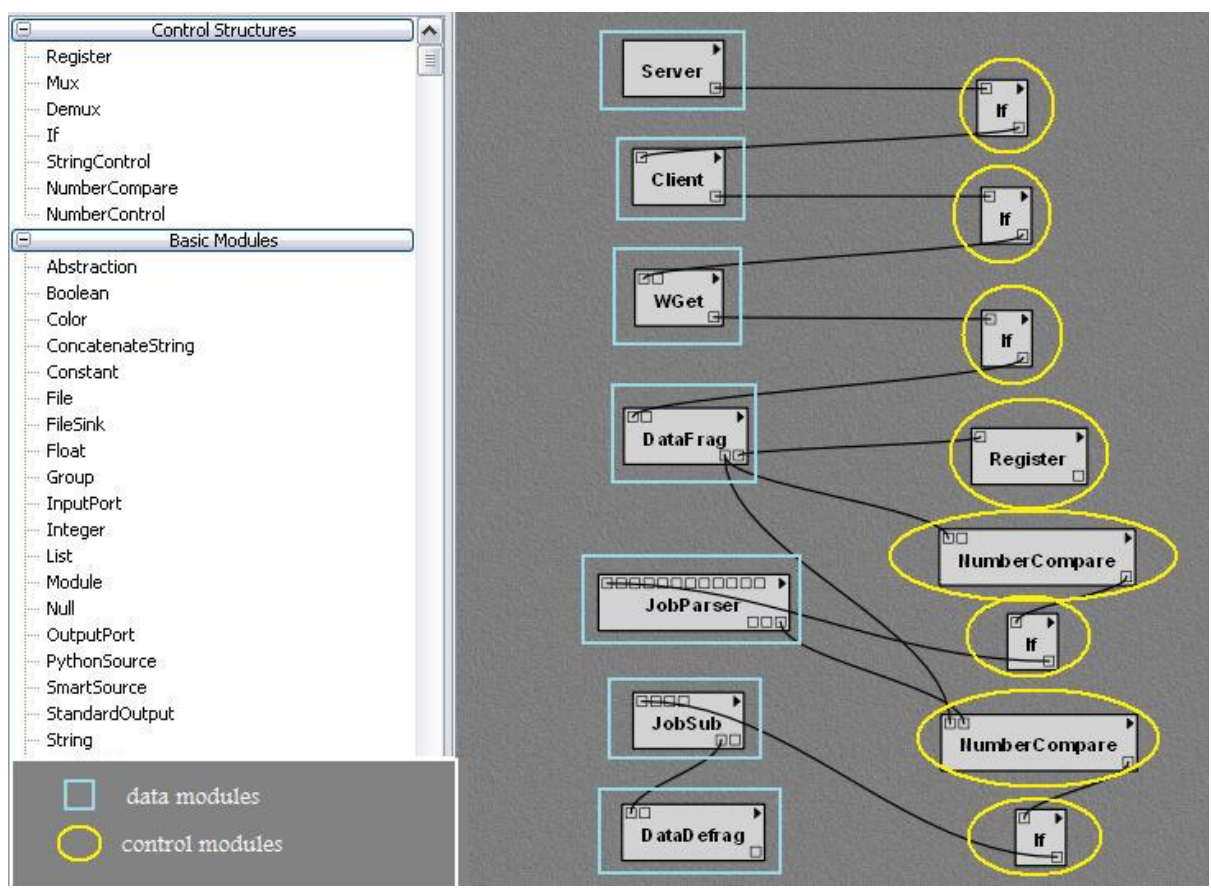

Fig. 2. Execution control modules on VisTrails. The highlighted processes in the left side (squares) are data intensive modules, from the MidMon middleware; the ones in the right side (ellipses) are control modules, from the "Control Structures" package.

MidMon is a lightweight middleware that presents a small number of structures to monitor the distributed execution of scientific workflow tasks. It was implemented with monitoring instrumentation middleware requirements in mind [19], providing a loosely coupled form of interaction with the WfMS. MidMon was designed as a multi-layer software, (scientists desktop and distributed environment layers) aiming to provide ease of use for scientific workflow developers, such as: being easily connected to a WfMS and monitor parallel applications; causing a negligible overhead on processing nodes and finally, taking advantage of remote machine job scheduling properties.

At the scientist desktop layer, the Server Monitor component allows scientists to monitor the status of distributed applications jobs. It receives unidirectional asynchronous monitoring messages submitted by Client Monitors on the distributed environment layer. The components of the distributed environment layer provide the ability for an execution thread to be diverged into several concurrent threads on a selective basis. Decoupling of monitors can allow greater scalability, lower message overheads and may be used on different network topologies.

In this work we redesigned the bioinformatics workflow using our control modules and MidMon modules to be executed using the extended VisTrails WfMS. The native VisTrails modules and our control-flow modules were coded in Python 2.5 and the MidMon Monitors in Java, so that new modules could be added to the workflow 
without major concerns. Unfortunately, VisTrails have no facilities to connect to distributed environments. Thus, in order to take advantage of its provenance gathering mechanisms it was also necessary to develop a module that enables remote connections to distributed environments. For the sake of simplicity, remote call modules were not represented in Figure 2. In our prototype, the BLAST package was represented as a one-step activity of the workflow and MidMon parallelized it.

Figure 2 shows MidMon modules inserted on the workflow design together with execution control modules in VisTrails. In short, the workflow runs as following. Initially, the Server Monitor is locally initiated. It receives asynchronous messages from the remote Client Monitor. Then, the Client Monitor is activated, to publish information's about remote processes and monitoring them. Considering the connection's establishment between them, if the server finds difficulty to be started, the Client Monitor cannot be activated, and the workflow must be finished. So, an If structure can be added between both modules; if everything goes well, the workflow continues; if not, it will be ended. After the Client Monitor, another If structure can be introduced, because if a connection error was found, and the Client Monitor could not be activated, for example, the service cannot keep on.

The same approach can be done between the WGet and DataFrag. The WGet module connects through the Internet to download the required database file. If the module successfully downloads it, the service can go on; nevertheless, if an error condition is reached (e.g. the downloaded file is corrupted), the workflow must be finished. DataFrag module divides the file in little fragments that will run in parallel on the distributed environment. In this part of the workflow, a NumberCompare module was added in order to compare the number of fragments created with the user-defined number of fragments. After this, an If structure was introduced; it will check if both quantities of fragments were the same. The DataFrag module also produces log information that can be visualized inside VisTrails, through the Spreadsheet window. In order to store this information and make it accessible and usable later, we added a Register module (used in the section 3.1.4) after the DataFrag. In this workflow, the flexibility of the control structures can be confirmed, once it was not necessary to change the structure of the DataFrag module to control the fragments.

The JobParser module generates, for each fragment, a parallel job that will be submitted to the distributed environment by the JobSub module. Between the JobParser and the JobSub modules, another NumberCompare and If modules can be added; the former compares the number of fragments created by DataFrag with the number of jobs produced; the If enables the WfMS to monitor remote errors, more specifically, one job, for some reason, was not produced; so, it can finish the execution if some jobs were not created.

\section{Conclusion}

Workflow technology and distributed environments provide the keystone to leverage the continuing evolution of e-Science. In order to fully explore opportunities provided by these paradigms, it is important to track and monitor experiments to gain new insight about the scientific experiment. In this paper, it was presented a set of generic control-flow modules that can extend workflow design power by providing dynamic 
behavioral features. Control-flow modules allow scientists to use some of the workflow patterns described by Aalst [15] [16] by adding these modules on the scientific workflow specification to be executed by heterogeneous WfMS running on distributed environments.

The proposed control-flow modules can be plugged into the workflow during design-time, helping scientists to face challenges of distributed workflow development. First, it acts as documentation of the execution control workflow behavior, recording in a concise and explicit fashion the operational characteristics of the workflow. Second, by adding control-flow facilities to a distributed monitoring middleware it is possible not only to evaluate and monitor the activities of the workflow but also gather provenance from heterogeneous and independent environments with low programming efforts.

Our work shares the same generic motivation of Groth, Munroe, Miles and Moreau [20] that is supporting provenance in any type of execution environment. Groth et al. developed the "p-structure" in a way that provenance is created by actors within the workflow, independent from the workflow definition language and from the workflow execution engine. In a similar way, our proposed control-flow structures can be seen as control-flow actors within the workflow. These actors are independent from workflow engines. This is helpful when a local workflow needs to run a sub-workflow in a high performance environment, i.e., control specification does not need to change. Our work may be seen as complementary to the "p-structure" in a sense that these control structures can embed "p-structures" to record workflow decisions and iterations, in addition to its capturing of the actual causal connections according to execution.

Developing MidMon on top of VisTrails presented some additional advantages: it enabled scientists to monitor the submitted jobs status on their desktops and as byproducts it preserves workflows' original features. It also allowed scientists to use a local centralized WfMS to connect and enact distributed activities, preserving the built-in facilities of provenance gathering. In addition, MidMon also complemented VisTrails tracking facilities, once VisTrails was not able to capture information about temporary and remote files created during distributed workflow execution.

By plugging these modules along the workflow specification we address the two challenges discussed at the motivation of this work. Using the control modules, design becomes less dependent of the WfMS specification language. Remote monitoring modules provide for parallel execution control in heterogeneous environments. Finally, these modules are the means of provenance gathering within a representation model that can be independent of the environment and the WfMS. The limitation of this approach is a verbose workflow specification due to several control processes that are not part of the scientific application domain. However, workflow view techniques [21] can hide these modules from the scientist. We are working on software component reuse techniques to help the automatic incorporation of these modules. Particularly in VisTrails, a "by example" tool for workflow design generation is under development. We intend to adapt it to recommend control modules that can help on workflow design, control and provenance gathering. Currently, other bioinformatics workflows are being analyzed to take advantage of MidMon combined to the proposed control-flow modules within VisTrails. 


\section{Acknowledgments}

This work was partially funded by CNPq. Chirigati and Dahis are supported by $\mathrm{CNPq}$ and the UFRJ/PIBIC program.

\section{References}

1. Freire, J., Koop, D., Santos, E., Silva, C.T.: Provenance for Computational Tasks: A Survey. In: Computing in Science and Engineering, pp. 20-30 (May/June 2008)

2. Gil, Y., Deelman, E., Ellisman, M., Fahringer, T., Fox, G., Gannon, D., Goble, C., Livny, M., Moreau, L., Myers, J.: Examining the challenges of scientific workflows. IEEE Computer 40(12), 26-34 (2007)

3. Yu, J., Buyya, R.: A taxonomy of scientific workflow systems for grid computing. ACM SIGMOD 34, 44-49 (2005)

4. Moreau, L., Freire, J., Futrelle, J., McGrath, R., Myers, J., Paulson, P.: The Open Provenance Model, Technical Report 14979, ECS EPrints repository (2007)

5. Bowers, S., Ludascher, B., Ngu, A.H.H., Critchlow, T.: Enabling Scientific Workflow Reuse through Structured Composition of Dataflow and Control-Flow. In: ICDE Workshops, pp. 70-80 (2006)

6. Goderis, A., Brooks, C., Altintas, I., Lee, E.A., Goble, C.: Composing Different Models of Computation in Kepler and Ptolemy II. In: Proc. of the 2nd Int. Workshop on Workflow Systems in e-Science (WSES 2007) in conjunction with the Int. Conference on Computational Science (ICCS) 2007, Beijing, China, pp. 27-30 (2007)

7. Tudruj, M., Kopanski, D., Borkowski, J.: Dynamic Workflow Control with Global States Monitoring. In: ISPDC 2007, Hagenberg, Austria, July 5-8 (2007)

8. Deelman, E., et al.: Pegasus: Mapping Scientific Workflows onto the Grid. In: AGC 2004, Cyprus (2004)

9. Kacksuc, P., Farkas, Z., Sipos, G., et al.: Workflow-level parameters study management in multi-Grid environments by P-GRADE Grid portal. In: GC 2006 Workshop at SC (2006)

10. Churches, D., et al.: Programming Scientific and Distributed Workflow with Triana Services. Concurrency: Practice and Experience 18(10), 1021-1037 (2006)

11. Hull, D., Wolstencroft, K., Stevens, R., Goble, C., et al.: Taverna: a tool for building and running workflows of services. Nucleic Acids Research 34 (Web Server issue), 729-732 (2006)

12. Callahan, S., Freire, J., Santos, E., Scheidegger, C., Silva, C., Vo, H.: VisTrails: visualization meets data management. In: ACM SIGMOD, pp. 745-747 (2006)

13. Scheidegeer, C., Koop, D., Santos, E., Vo, H., Callahan, S., Freire, J., Silva, C.: Tackling the Provenance Challenge One Layer at a Time. Concurrency and Computation: Practice and Experience 20(5), 473-483 (2007)

14. Ludäscher, B., et al.: Scientific workflow management and the Kepler system. Concurrency and Computation: Practice and Experience 18(10), 1039-1065 (2006)

15. Russell, N., Hofstede, A., ter, A.W.M.P., van der Mulyar, N.: Workflow Control-Flow Patterns: A Revised View. Technical Report BPM-06-22, BPM Center (2006)

16. Aalst, W.M.P., van der Hosftede, A.H.M., ter Kiepuszewski, B., Barros, A.P.: Workflow Patterns. Distributed and Parallel Databases 14(1), 5-51 (2003)

17. Goble, C., Wroe, C., Stevens, R.: The myGrid project: services, architecture and demonstrator. In: UK e-Science All Hands Meeting (2003) 
18. Cruz, S.M.S., Silva, F.N., Gadelha Jr., L., Cavalcanti, M., Campos, M., Mattoso, M.: A Lightweight Middleware Monitor for Distributed Scientific Workflows. In: 3rd WSES/CCGrid (2008)

19. Truong, H., Fahringer, T., Dustdar, S.: Dynamic Instrumentation, Performance Monitoring and Analysis of Grid Scientific Workflows. Journal of Grid Computing 3, 1-18 (2005)

20. Groth, P., Munroe, S., Miles, S., Moreau, L.: HPC and Grids in Action. In: Grandinetti, L. (ed.) Applying the Provenance Data Model to a Bioinformatics Case. IOS Press, Amsterdam (2008)

21. Davidson, S., Cohen-Boulakia, S., Eyal, A., Ludaescher, B., McPhillips, T., Bowers, S., Anand, M., Freire, J.: Provenance in Scientific Workflow Systems. IEEE Data Eng. Bull. 30(4), 44-50 (2007) 